\title{
Clinicopathologic characteristics and prognostic factors for primary spinal epidural lymphoma: report on 36 Chinese patients and review of the literature
}

\author{
Le Xiong $^{1+}$, Ling-Min Liao ${ }^{2 \dagger}$, Jian-Wu Ding ${ }^{1}$, Zhi-Lin Zhang ${ }^{3}$, An-Wen Liu ${ }^{1 *}$ and Long Huang ${ }^{1 *}$
}

\begin{abstract}
Background: Due to the uncommon nature of primary spinal epidural lymphomas (PSELs), there has been little research looking at prognostic indicators for the tumor. To our knowledge, this is the largest study to evaluate possible clinical and pathologic prognostic factors in PSEL patients.

Methods: We retrospectively reviewed 130 cases of PSEL, including 36 Chinese patients and 94 published case reports from 1985 to 2015. Patient treatment regimens included surgery $(S ; n=119)$, surgery followed by chemotherapy $(S+C T ; n=25)$, surgery followed by radiotherapy $(S+R T ; n=26)$, and surgery followed by chemotherapy and radiotherapy $(S+C T+R T ; n=50)$.

Results: Review of the most recent case follow-up data (time varied) found 51 patients (47\%) alive and tumorfree, 10 patients (9\%) alive with tumor present, and 47 patients (44\%) deceased. The 3-year overall survival (OS) and disease-free survival (DFS) rates were $81.1 \%$ and $46.3 \%$, respectively. Favorable prognostic factors found by univariate analysis were female sex, B-cell lymphoma diagnosis, cervical spine location, and combined modality treatment. Furthermore, multivariate analysis revealed that thoracic spine location $(\mathrm{HR}=4.629,95 \% \mathrm{Cl}=[1.911$, 31.667], $P=0.042$ for OS) and the lack of combined modality treatment ( $\mathrm{HR}=12.697,95 \% \mathrm{Cl}=[2.664,48.612]$, $P<0.0001$ for DFS) were associated with poor survival in PSEL patients.

Conclusions: PSEL demonstrates specific clinical features and is associated with a relatively good prognosis. Thoracic spine location is a significant poor prognostic factor, and combined modality treatment is associated with improved disease-free survival, but not overall survival.
\end{abstract}

Keywords: Primary spinal epidural lymphomas, Treatment, Prognosis

\section{Background}

Primary spinal epidural lymphomas (PSELs) are very rare, and relatively few such tumors have been reported in the literature. Although PSEL accounts for $10 \%$ of all epidural spinal tumors [1], the epidural location is a rare site of presentation for lymphomas, characterizing only 0.1 to $3.3 \%$ of all lymphomas [2,3]. Due to the rarity of the condition, it is difficult to enroll a sufficient number

\footnotetext{
*Correspondence: awliu666@163.com; huanglongdoctor@163.com ${ }^{\dagger}$ Equal contributors

'Department of Oncology, The Second Affiliated Hospital of Nanchang University, 1 Minde Road, Nanchang, Jiangxi, China

Full list of author information is available at the end of the article
}

of patients with PSEL to adequately evaluate possible factors associated with survival. The largest series to date, reported by Monnard et al. [4], identified 52 patients over 20 years (1982-2002). However, the limited number of patients and the long time span have made it difficult to establish the disease parameters, such as the natural history of the disease, prognostic indicators, treatment techniques employed, and survival rates. For this study, we identified only retrospective studies, which included reports on relatively small numbers of patients or single case reports. The goal of this study was to help identify the clinical profile, treatment outcomes, and significant prognostic indicators in PSEL patients. 


\section{Methods}

\section{Patient characteristics}

A total of 130 PSEL cases were retrospectively reviewed, including 36 Chinese patients and 94 published case reports from 1985 to 2015. Patients were selected if there was a conclusive histopathologic diagnosis of PSEL with complete clinical pathology, and there was no history of previous malignant disease or a second primary tumor. The 130 patients included 76 men (58\%), 44 women (34\%) and 10 unknown (8\%), for a known male-to-female ratio of 1.73 . The median age was 45 years (range, $3-80$ years), and median follow-up was 32 months (range, 1-149 months). Clinical and pathological variables analyzed included patient age and gender; tumor stage, differentiation, location, and range; and treatment modalities employed (Table 1).

\section{Treatment}

Most patients diagnosed with stage IE-IIE PSEL had been treated with tumorectomy $(n=121,93 \%)$; the rest had surgical contraindications or refused surgery. The primary treatment of patients consisted primarily of surgery. Individualized postoperative treatment consisted of radiation therapy alone $(n=26,21 \%)$, chemotherapy alone $(n=20,25 \%)$, or concurrent chemoradiation therapy $(n=41,49 \%)$. The median radiotherapy dose was 40 Gy (range, 20-50 Gy), and the total dose was administered over 3-5 weeks. Twenty-eight patients (34\%) had only focal treatment, whereas the remaining 55 (66\%) received more than focal treatment. Eight-two patients (63\%) had chemotherapy, which consisted of cyclophosphamide, doxorubicin, vincristine, and prednisone in the majority of patients; methotrexate, leucovorin, etoposide, and bleomycin were also used in some patients. Generally, 4-8 cycles of chemotherapy were administered at 3-week intervals. Methotrexate was administered in 21 patients (26\%).

\section{Statistical analysis}

The Kaplan-Meier method and log-rank tests were used to determine overall survival (OS) and disease-free survival (DFS). Independent prognostic factors for OS and DFS were identified using the Cox proportional hazards model. SPSS software (version 13.0, SPSS Inc., Chicago, IL) was used for all statistical analyses, and $P$ values $<0.05$ were considered statistically significant.

\section{Results}

\section{Clinical features}

The median patient age was 45 years (range, $3-80$ years). In three of the 130 patients (2\%), PSEL was detected during a routine examination. Alternatively, 112 of the patients (86\%) presented with neck, back, lumbosacral, or limb pain, or with cord compression syndrome. For 15
Table 1 Analysis of clinicopathological factors for chinese patients and report cases

\begin{tabular}{|c|c|c|c|}
\hline \multirow[t]{2}{*}{ Characteristics } & \multirow{2}{*}{$\begin{array}{l}\text { Chinese patients } \\
n=36(\%)\end{array}$} & \multirow{2}{*}{$\begin{array}{l}\text { Report cases } \\
n=94(\%)\end{array}$} & \multirow[t]{2}{*}{$P$ value } \\
\hline & & & \\
\hline Median age (year) & 26 & 48 & / \\
\hline Median survival (month) & 12 & 27 & / \\
\hline \multicolumn{4}{|l|}{ Gender } \\
\hline Male & $26(74.3)$ & $50(60.0)$ & \\
\hline Female & $9(25.7)$ & $34(40.0)$ & 0.640 \\
\hline \multicolumn{4}{|l|}{ Pathology } \\
\hline B cell lymphoma & $25(78.1)$ & $69(74.2)$ & \\
\hline T cell lymphoma & $6(18.8)$ & $7(7.5)$ & \\
\hline Burkitt's lymphoma & $1(3.1)$ & $17(18.3)$ & 0.035 \\
\hline \multicolumn{4}{|l|}{ Stage } \\
\hline । & $13(76.5)$ & $44(81.5)$ & \\
\hline II-IV & $4(23.5)$ & $10(18.5)$ & 0.651 \\
\hline \multicolumn{4}{|l|}{ Range } \\
\hline 1-2 spine & $8(22.9)$ & $25(50.0)$ & \\
\hline 3-4 spine & $21(60.0)$ & $14(28.0)$ & \\
\hline$\geq 5$ spine & $6(17.1)$ & $11(22.0)$ & 0.010 \\
\hline \multicolumn{4}{|l|}{$S+R T+C T$} \\
\hline Yes & $5(14.3)$ & $45(48.9)$ & \\
\hline No & $30(85.7)$ & $47(51.1)$ & $<0.001$ \\
\hline \multicolumn{4}{|l|}{$S+C T$} \\
\hline Yes & $17(56.7)$ & $57(62.0)$ & \\
\hline No & $18(43.3)$ & $35(38.0)$ & 0.172 \\
\hline \multicolumn{4}{|l|}{ S } \\
\hline Yes & $33(94.3)$ & $85(92.4)$ & \\
\hline No & $2(5.7)$ & $7(7.6)$ & 0.710 \\
\hline \multicolumn{4}{|l|}{ S only } \\
\hline Yes & $15(42.9)$ & $3(3.3)$ & \\
\hline No & $20(57.1)$ & $89(96.7)$ & $<0.001$ \\
\hline
\end{tabular}

cases (12\%) reviewed from published case reports, the details of presentation were not provided. The most commonly reported symptoms were motor weakness or hypoesthesia (62\%), back pain (59\%), lumbosacral pain (32\%), limb pain (28\%), neck pain (9\%), bowel dysfunction (23\%), bladder dysfunction (19\%), and low-grade fevers (2\%). The time from the first symptom to diagnosis varied from 3 days to 5 years. Patient characteristics are presented in Additional file 1: Table S1 and Additional file 2: Table S2.

\section{Pathologic features}

Case reviews showed tumor pathologies of B-cell lymphoma $(n=88,76 \%)$, T-cell lymphoma $(n=13,11 \%)$, or Burkitt's lymphoma $(n=15,13 \%)$. Subsets of cases were 
identified with tumor cells showing the strong expression of leukocyte common antigen $(19 / 19,100 \%)$ or the absence of CD20 expression (21/27, 78\%). Additional immunohistochemical assays performed in a subset of the cases included detection of CD3 (8/13, 62\%), CD45 (6/9, 67\%), and CD79 (7/7, 100\%). See Additional file 1: Table S1 and Additional file 2: Table S2.

\section{Survival}

The median follow-up period was 32 months. Relapses were observed in 21 patients (19\%) after a median period of 12 months, primarily in the central nervous system $(n=11)$, lymph nodes $(n=4)$, chest or abdomen $(n=5)$, and liver $(n=2)$. The 3 -year OS and DFS were 81.1 and $46.3 \%$, respectively.

\section{Prognostic factors}

In order to identify potential prognostic factors associated with survival in PSEL patients, various clinicopathologic variables were evaluated (Table 2). Using univariate analysis, gender, pathological pattern, tumor differentiation, tumor location, and tumor range were found to be associated with OS $(P<0.05)$, but not with DFS. The 3year OS rates for males and females were $60.3 \%$ and $100 \%(P=0.002)$, respectively (Fig. 1a). The 3-year OS rates for thoracic, cervical, and lumbosacral spine locations were $64.1,94.3$, and $100 \%(P=0.005)$, respectively (Fig. 1b). The 3-year OS rates for B-cell, T-cell, and Burkitt's lymphoma were 87.7, 83.3, and $29.5 \%(P=$ 0.002 ), respectively (Fig. 1c). In terms of treatments received, the 3-year DFS rate for patients receiving $S$ alone was $23.3 \%$ ( $P=0.004$, compared with the other treatment groups; Fig. 2), while patients receiving $\mathrm{S}+\mathrm{RT}+\mathrm{CT}$ had a 3 -year DFS rate of $49.6 \%(P=0.031$, compared with the other treatment groups, Fig. 3); patients receiving $\mathrm{S}+\mathrm{CT}$ had a 3-year DFS rate of 50.4\% $(P=0.042$, compared with the other treatment groups; Fig. 4). For the same patient treatment groups, the 3-year OS rates were 80.0, 81.7, and $79.5 \%$, respectively; these did not significantly vary $(P>$ 0.05 for all; Figs. 5, 6, and 7). Multivariate analysis revealed that thoracic spine location $(\mathrm{HR}=4.629,95 \% \mathrm{CI}=[1.911$, 31.667], $P=0.042$ for OS) and the lack of combined modality treatment $(\mathrm{HR}=12.697,95 \% \mathrm{CI}=[2.664,48.612], P$ $<0.0001$ for DFS) were associated with poor survival in PSEL patients.

\section{Discussion}

With a study cohort of 130 patients treated over a 30year period, the current study represents the largest one to focus on PSEL. Prior to this, the largest one reported on only 52 patients, and predated modern radiotherapy and chemotherapy protocols.

The origin of primary spinal epidural lymphomas (PSEL) remains controversial. It is known that lymphoma
Table 2 The 3-year' OS and DFS rates associated with primary spinal epidural lymphomas patients

\begin{tabular}{|c|c|c|c|c|c|}
\hline \multirow[t]{2}{*}{ Characteristics } & \multirow{2}{*}{$\begin{array}{l}\text { Case } \\
(n)\end{array}$} & \multicolumn{2}{|l|}{3 year's } & \multicolumn{2}{|c|}{3 year's } \\
\hline & & $\mathrm{DFS}(\%)$ & $\mathrm{P}$ & OS & $P$ \\
\hline \multicolumn{6}{|l|}{ Age (year) } \\
\hline$<45$ & 64 & 54.8 & & 70.5 & \\
\hline$\geq 45$ & 64 & 41.8 & 0.413 & 90.9 & 0.181 \\
\hline \multicolumn{6}{|l|}{ Gender } \\
\hline Male & 76 & 47.7 & & 60.3 & \\
\hline Female & 44 & 47.7 & 0.608 & 100.0 & 0.002 \\
\hline \multicolumn{6}{|l|}{ Pathology } \\
\hline B cell lymphoma & 88 & 47.6 & & 87.7 & \\
\hline T cell lymphoma & 13 & 53.6 & & 83.3 & \\
\hline Burkitt's lymphoma & 15 & 25.0 & 0.081 & 29.5 & 0.002 \\
\hline \multicolumn{6}{|l|}{ Differentiate } \\
\hline Poor & 19 & 54.5 & & 74.1 & \\
\hline Moderate & 12 & 50.5 & & 68.2 & \\
\hline High & 8 & 60.0 & 0.309 & 100.0 & 0.035 \\
\hline \multicolumn{6}{|l|}{ Stage } \\
\hline I & 58 & 36.7 & & 75.1 & \\
\hline II-IV & 14 & 77.4 & 0.084 & / & 0.198 \\
\hline \multicolumn{6}{|l|}{ Location } \\
\hline Cervical & 14 & 74.0 & & 94.3 & \\
\hline Thoracic & 69 & 35.7 & & 64.1 & \\
\hline Lumbosacral & 25 & 57.4 & & 100.0 & \\
\hline Sacral & 3 & / & 0.089 & / & 0.005 \\
\hline \multicolumn{6}{|l|}{ Range } \\
\hline 1-2 spine & 17 & 36.4 & & 86.2 & \\
\hline 3-4 spine & 33 & 46.7 & & 81.9 & \\
\hline$\geq 5$ spine & 18 & 41.2 & 0.698 & 74.4 & 0.013 \\
\hline \multicolumn{6}{|l|}{$\mathrm{S}+\mathrm{RT}+\mathrm{CT}$} \\
\hline Yes & 49 & 49.6 & & 81.7 & \\
\hline No & 56 & 38.0 & 0.031 & 81.4 & 0.955 \\
\hline \multicolumn{6}{|l|}{$S+C T$} \\
\hline Yes & 65 & 50.4 & & 79.5 & \\
\hline No & 40 & 41.4 & 0.042 & 84.3 & 0.984 \\
\hline \multicolumn{6}{|l|}{$S$} \\
\hline Yes & 96 & 46.8 & & 80.0 & \\
\hline No & 9 & 47.6 & 0.994 & 100.0 & 0.592 \\
\hline \multicolumn{6}{|l|}{ S only } \\
\hline Yes & 5 & 23.3 & & 80.0 & \\
\hline No & 100 & 75.6 & 0.004 & 81.6 & 0.437 \\
\hline
\end{tabular}

may arise anywhere lymphatic tissue is found. However, whether there is lymphoid tissue in the epidural space has been debated $[5,6]$. Rubinstein was the first to 

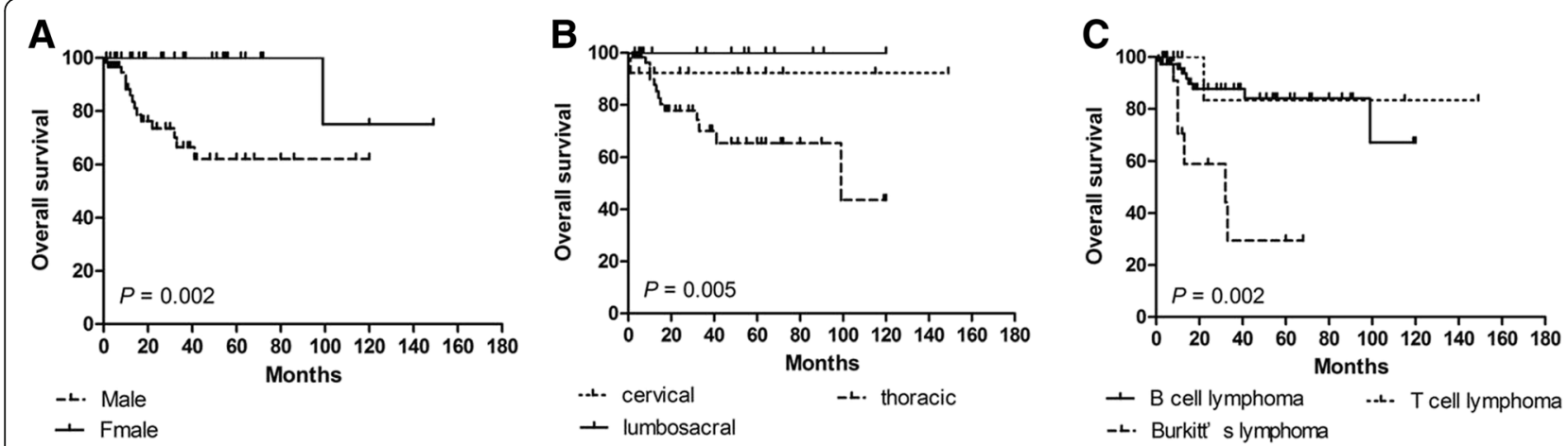

Fig. 1 Kaplan-Meier survival curves for clinicopathologic factors of patients with primary spinal epidural lymphomas. a Survival curves for OS in relation to gender in primary spinal epidural lymphomas as indicated. b Survival curves for OS in relation to tumor location. c Survival curves for OS associated with different pathological factors as indicated. Favorable prognostic factors in primary spinal epidural lymphoma patients were female sex, B-cell lymphoma type, and cervical spine location

demonstrate the presence of lymphoid cells in epidural tissue, and he introduced the theory of antigenic stimulation with a transformation cascade [7]. Some have suggested that PSEL may originate from either paraspinal, spinal, or retroperitoneal tissues, accessing the epidural space via the interspinal foramina $[8,9]$. However, the occurrence of lymphoma in this location indicates that lymphoid precursor cells are present in the space. Metastasis is the most common sacral malignancy, whereas chordoma in the most common primary sacral tumor [10]. PSELs represent $10 \%$ of epidural spinal tumors, but the epidural location is a rare presenting site for lymphomas, being seen in only 0.1 to $3.3 \%$ of all lymphomas. When lymphoma has been found in the spine, it has been reported most often in the lumbar or lower dorsal area [11]. However, in this study, we found the thoracic spine to be the most common site (62\%) for lymphoma.

The most common presenting symptoms reported in the literature have included lower limb weakness, localized back pain, and bladder dysfunction [8, 9]. However, in this study, the most common symptoms seen were motor weakness or hypoesthesia (62\%), back pain (59\%), lumbosacral pain (32\%), limb pain (28\%), neck pain (9\%), bowel dysfunction (23\%), bladder dysfunction (19\%), and low-grade fevers (2\%). It is noteworthy that most patients presented with persistent back pain for considerably long periods of time prior to diagnosis. Therefore, persistent back pain in a patient should be considered a warning symptom for a more serious illness.

On histopathological examination, these tumors show atypical lymphoid cell proliferation. On immunohistochemistry, tumor cells are positive for leukocyte common antigen (LCA) and CD20, but negative for CD138, CD30, and CD3. B-cell lymphoma is the most common type at this site [12], which is consistent with our findings. Our study found that tumor pathologies consisted of B-cell lymphoma (76\%), T-cell lymphoma (11\%), and Burkitt's lymphoma (13\%). Furthermore, tumor cells were associated with the strong expression of leukocyte

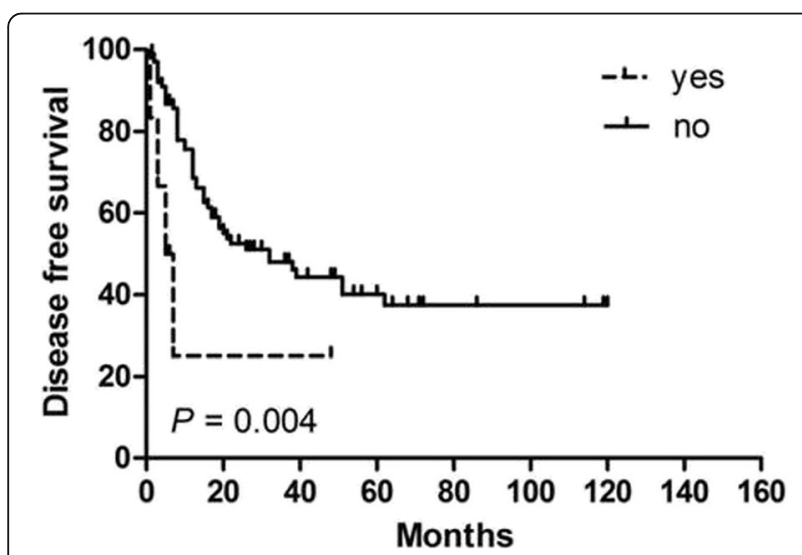

Fig. 2 Kaplan-Meier survival curves analyze for DFS rates associated with surgery alone vs. others



Fig. 3 Kaplan-Meier survival curves analyze for DFS rates associated with surgery followed by chemotherapy and radiotherapy vs. others 


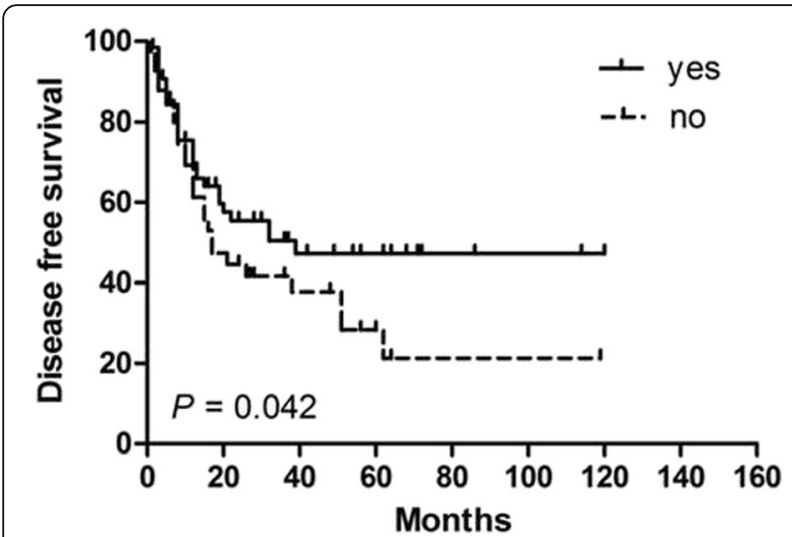

Fig. 4 Kaplan-Meier survival curves analyze for DFS rates associated with surgery followed by chemotherapy vs. others

common antigen (100\%) and the absence of CD20 expression $(78 \%)$. PSELs are almost invariably of the B-cell type, although indolent $\mathrm{B}$-cell and $\mathrm{T}$-cell variants are sometimes seen.

There has been no optimization of treatment strategies for PSEL patients to improve patient outcomes. Unfortunately, there is a high mortality rate within the first year of diagnosis due to early metastasis and tumor recurrences. Accordingly, the importance of aggressive, systemic therapy has been recognized over the last few decades, although there have been reports of patients being cured with local radiotherapy alone [13]. In cases of patients who present with signs of spinal cord compression, surgical intervention for tissue diagnosis and decompression is required. Aabo et al. [14] reported no difference between the outcomes in patients who underwent decompressive laminectomy and radiotherapy and those who received spinal radiation only. Chemoradiotherapy remains the mainstay of treatment because lymphomas are highly radio- and chemosensitive tumors.

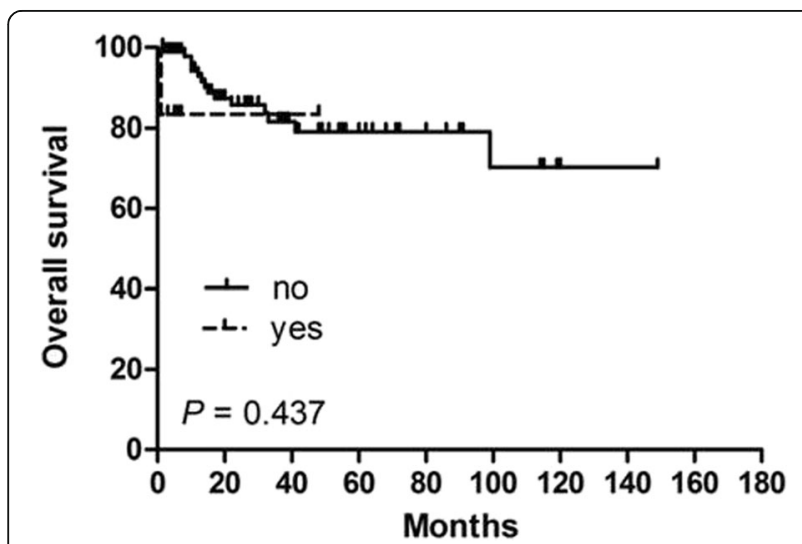

Fig. 5 Kaplan-Meier survival curves analyze for OS rates associated with surgery alone vs. Others

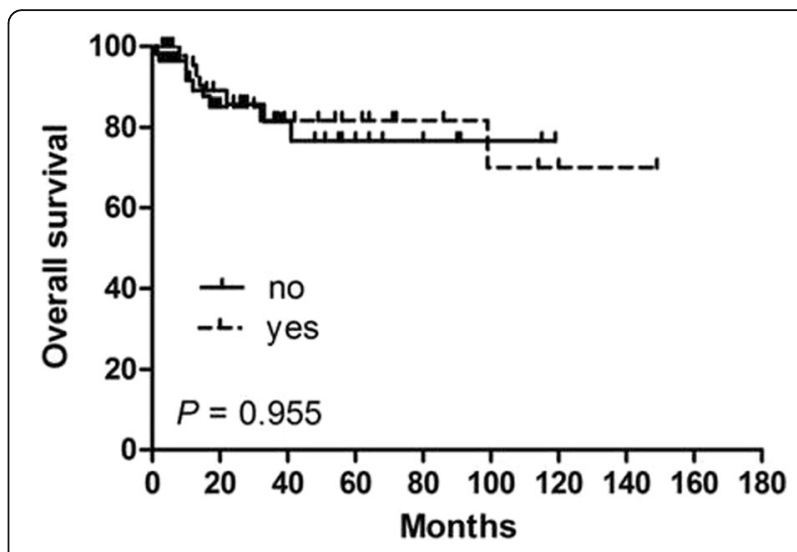

Fig. 6 Kaplan-Meier survival curves analyze for OS rates associated with surgery followed by chemotherapy and radiotherapy vs. others

The CHOP regime, in particular, is still considered the gold standard for treatment. A radiotherapy dose of 3500 cGy to 4000 cGy delivered in 20-25 fractions over 3-4 weeks is required to achieve radical cure [9]. Intensive chemotherapy should also be initiated immediately following diagnosis and be delivered over a short period of time. From reviewing several studies, we found that combined modality treatment is associated with a lower recurrence rate and improved disease-free survival in PSEL, but not overall survival. Similar findings have not been reported by other researchers. In this study, the median survival time of 36 Chinese patients is 12 month. It shorter than non-Chinese cases (12 VS. 27). Optimization of treatment strategies for Chinese patients included surgery (33/35, $94.3 \%)$, surgery followed by chemotherapy (17/35, 56.7\%), surgery only $(15 / 35,42.9 \%)$, and surgery followed by chemotherapy and radiotherapy $(5 / 35,14.3 \%)$. There are less Chinese patients with PSEL treated with combined modality treatment compared to non-Chinese cases because of medical expenses. Kaplan-Meier

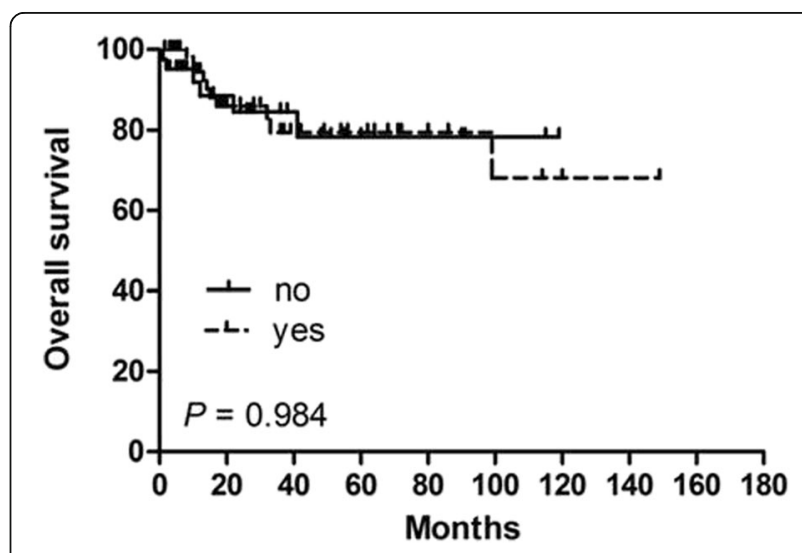

Fig. 7 Kaplan-Meier survival curves analyze for OS rates associated with surgery followed by chemotherapy vs. others 
analysis revealed that combined modality treatment is associated with improved disease-free survival for all 130 PSEL cases. Therefore, to our knowledge, combined modality treatment should be considered an standardized treatment strategy for PSEL presently.

\section{Conclusions}

The results of the present study indicate that PSEL has a relatively good prognosis, that thoracic spine location is a significant poor prognostic factor, and that combined modality treatment is associated with improved diseasefree survival, but not overall survival. This study represents the largest to date on PSEL although it is limited by its retrospective design and the relatively small patient cohort. Nevertheless, our findings give important insight into this rare, challenging disease, and expand our knowledge base on this aggressive tumor.

\section{Additional files}

Additional file 1: Table S1. The clinical and pathologic features of Primary spinal epidural lymphomas cases examined in China. (XLSX 13 kb)

Additional file 2: Table S2. The clinical and pathologic features of Primary spinal epidural lymphomas cases published. (XLSX $20 \mathrm{~kb}$ )

\section{Abbreviations}

Cl: Confidence interval; CT: Chemotherapy; DFS: Disease-free survival; HR: Hazard ratio; OS: Overall survival; PSELs: Spinal epidural lymphomas; RT: Radiotherapy; S: Surgery

\section{Acknowledgements}

Not applicable.

\section{Funding}

This work was supported by the National Natural Science Foundation of China [grant numbers 81460393], the Natural Science Foundation of Jiangxi Province, China [grant numbers 20142BAB215039, 20151BAB215020], the Project of Jiangxi Province Science and Technology Plan [grant number 20141BBG70041], the Project of Education Department of Jiangxi Province Science and Technology Plan [grant number GJJ14059], the Youth Science Fund Project of the Second Affiliated Hospital of Nanchang University [grant number 2014YNQN12004 to Long Huang], and supported in part by the Natural Science Foundation of China [grant number 81460449 to Ling-Min Liao]. The Grants-in-Aid supported this study just financially, and was not associated with study design, collection, analysis, interpretation of data, and writing the manuscript.

\section{Availability of data and materials}

The data supporting our findings was presented within additional supporting files.

\section{Authors' contributions \\ $L X$ and $L M L$ participated in the study design, case collection, drafting, and revising the manuscript. JWD participated in the study design and revising the manuscript. ZLZ participated in the case collection. AWL and LH conceived the study, was responsible for its design and coordination, participated in the analysis and interpretation of the data, as well as in drafting and revising all versions of the manuscript. All authors read and approved the final manuscript.}

\section{Competing interests}

The authors declare that they have no competing interest.

\section{Ethics approval and consent to participate}

This study was approved by the ethics committee of the Second Affiliated Hospital of Nanchang University. Authors obtained written informed consent and publication consent from the participants or their guardian in the case of children under 16.

\section{Author details}

${ }^{1}$ Department of Oncology, The Second Affiliated Hospital of Nanchang University, 1 Minde Road, Nanchang, Jiangxi, China. ${ }^{2}$ Department of Ultrasound, The Second Affiliated Hospital of Nanchang University, Nanchang, China. ${ }^{3}$ Department of Otorhinolaryngology, The Second Affiliated Hospital of Nanchang University, Nanchang, China.

Received: 12 September 2016 Accepted: 27 January 2017

Published online: 14 February 2017

\section{References}

1. Boukobza M, Mazel C, Touboul E. Primary spinal and spinal epidural nonHodgkin's lymphoma with spinal cord compression. Neuroradiology. 1996; 38:333-7.

2. Salvati M, Cervoni L, Artico M, Raco A, Ciappetta P, Delfini R. Primary spinal epidural non-Hodgkin's lymphomas: a clinical study. Surg Neurol. 1996;46: 339-44.

3. Lim CCT, Chong BK. Spinal epidural non-Hodgkin's lymphoma: Case reports of three patients presenting with spinal cord compression. Singapore Med J. 1996:37:497-500.

4. Monnard V, Sun A, Epelbaum R, Poortmans P, Miller RC, Verschueren T, et al. Primary spinal epidural lymphoma: patients' profile, outcome, and prognostic factors: a multicenter Rare Cancer Network study. Int J Radiat Oncol Biol Phys. 2006;65:817-23.

5. Lyons MK, O'Neill BP, Kurtin PJ, Marsh WR. Spinal epidural non-Hodgkin's lymphoma. Mayo Clin Proc. 1996;71:453-7.

6. Grant JW, Kaech D, Jones DB. Spinal cord compression as the first presentation of lymphoma-a review of 15 cases. Histopathology. 1996;10:1191.

7. Rubinstein $\sqcup$. Tumor of the nervous system. Arch of tumor pathology. Facide 6. Tumor of the lymphorect System. AFIP. 1972;66:13.

8. Mclain RF, Markman M. Cancer in the spine: comprehensive care. Totowa: Human Press; 2006. p. 111.

9. Kapoor R, Kumar V, Sharma SC. Primary extradural non-Hodgkin's lymphoma. JK Science. 2006;8:45-8.

10. Liu JK, Kan P, Schmidt MH. Diffuse large B-cell lymphoma presenting as a sacral tumor. Report of two cases. Neurosurg Focus. 2003;15:1-5.

11. Haddad P, Thaell JF, Kiely JM, Harrison EG, Miller RH. Lymphoma of the spinal extradural space. Cancer. 1976:38:1862-6.

12. McDonald AC, Nicoll JA, Rampling RP. Non-Hodgkin's lymphoma presenting with spinal cord compression: a clinicopathological review of 25 cases. Eur J Cancer. 2000;36:207-13.

13. Wada N, Kohara M, Ikeda J, Hori Y, Fujita S, Okada M, et al. Diffuse large Bcell lymphoma in the spinal epidural space: a study of the Osaka Lymphoma Study Group. Pathol Res Pract. 2010;206:439-44.

14. Aabo K, Walbom-Jrgensen S. Central nervous system complications by malignant lymphomas: radiation schedule and treatment results. Int J Radiat Oncol Biol Phys. 1986;12:197-202.

Submit your next manuscript to BioMed Central and we will help you at every step:

- We accept pre-submission inquiries

- Our selector tool helps you to find the most relevant journal

- We provide round the clock customer support

- Convenient online submission

- Thorough peer review

- Inclusion in PubMed and all major indexing services

- Maximum visibility for your research

Submit your manuscript at www.biomedcentral.com/submit 